\title{
Frontières
}

\section{L'anticipation pure}

\section{L'annonce de la mort}

\section{Christian Saint-Germain}

Volume 14, numéro 2, printemps 2002

La mort prononcée

URI : https://id.erudit.org/iderudit/1073982ar

DOI : https://doi.org/10.7202/1073982ar

Aller au sommaire du numéro

Éditeur(s)

Université du Québec à Montréal

ISSN

1180-3479 (imprimé)

1916-0976 (numérique)

Découvrir la revue

Citer ce document

Saint-Germain, C. (2002). L'anticipation pure : l'annonce de la mort. Frontières, 14(2), 93-94. https://doi.org/10.7202/1073982ar

Ce document est protégé par la loi sur le droit d'auteur. L'utilisation des services d'Érudit (y compris la reproduction) est assujettie à sa politique d'utilisation que vous pouvez consulter en ligne.

https://apropos.erudit.org/fr/usagers/politique-dutilisation/
Cet article est diffusé et préservé par Érudit.

Érudit est un consortium interuniversitaire sans but lucratif composé de l’Université de Montréal, l'Université Laval et l'Université du Québec à Montréal. Il a pour mission la promotion et la valorisation de la recherche. https://www.erudit.org/fr/ 


\title{
L'anticipation pure L'ANNONCE DE LA MORT
}

\author{
« NOUS DONNONS FIGURE HUMAINE À L'ABÎME, EN L'APPELANT NAÎTRE ET MOURIR. »
}

(LEGENDRE, 1996, p. 12).

\section{Christian Saint-Germain, Ph.D., conseiller sur le thème.}

Le pari exorbitant de ce numéro de Frontières consistait à mettre en évidence, de façon descriptive surtout, certains signes par lesquels la mort s'annonce ou plus exactement, par lesquels elle nous est annoncée. Signes, symptômes, interceptés par celui ou celle qui va mourir, ou oraison pour ceux qui restent et qui signent dans ces sombres palabres l'étrange calligraphie d'un événement invraisemblable. Cela impliquait rien de moins que de penser une fumée sans feu ; comme si, à cette occasion, des signes ne cessaient de se montrer sans jamais se faire voir ou s'employaient à ne jamais laisser percevoir ce dont ils sont pourtant les indices indiscutables. L'étrangeté et le lointain se referment autour de celui ou celle qui en décèle l'apparence. Une sorte de «monstration» du monstrueux tapi survient dans une parole qui sidère ou abat, qui donne la mort désormais à attendre et à entendre. Nous réentendons les phrases des comptines maternelles: Au loup! Au loup! Alors que nous nous amusions à apprendre la peur.

La mort est donnée dans ce qui l'annonce. L'effroyable vient à travers la médiation du banal, fût-il savant ou ésotérique; il ne se présente pas en propre. Avec l'annonce de la mort, le mal est fait car cette épreuve ne permet pas le corps à corps valeureux avec ce qui s'avance, le menaçant, mais laisse chacun en pièces ou en souvenirs détachés. Notre propension est au vestige.

C'est cette inquiétante signalisation d'une fin abrupte ou la «chronique d'une mort annoncée» qui mérite d'être lue au revers d'une lame du tarot, à la réception des résultats d'une radiographie ou à la lecture consternante d'une résonance électro- magnétique. Mais comment l'apprendre à autrui, en communiquer l'intrigant verdict quand on est celui qui la voit - tout en symptômes précurseurs - et qui soupçonne sur ces entrefaites sa propre fin, comme la fin de celui ou celle qui se trouve éventuellement «condamné » par la médecine?

Nous avions voulu penser la simple apparition de ce qui jamais ne se manifeste complètement. Ce avec quoi, tout au cours de notre vie, nous n'en finissons jamais de finir. Penser ou désigner le site innommable d'un non-lieu est ici une seule et même chose, comme le plus terrible aboutissement de la chair. N'est-il pas paradoxal que la mort, ce qui nous est le plus propre, comme le centre de gravité des décisions de notre existence, ne nous arrive le plus souvent que sous la forme de l'annonce, colportée en quelque sorte, ou par personne interposée? Nous n'aurons jamais autre chose qu'une connaissance indirecte, par ouï-dire de ce phénomène.

La dette contractée avec la mort en naissant ne s'élucide qu'à la hauteur de la philosophie ou encore dans les plis prosaïques du temps salarié insignifiant. À cette intersection, le fait de tourner les pages grises d'un journal du métro ou de repenser au glissement sémantique entre échéance et déchéance dans les traductions de Être et Temps de Martin Heidegger permet de retrouver une signification à notre attente.

Nous apprenons - cette annonce - dans la rumeur des symptômes et des voix, mais aussi dans l'ordinaire des rendez-vous avec les représentants de l'institution médicale. Ainsi par exemple : le patient de la chambre 15 est mort ce matin. Autre situation commune : alors que la vie paraît continuer pour chacun qui s'affaire à sourire, à lire dans une salle d'attente bancale, un homme doit revenir chez lui conscient du fait que quelque chose d'irrémédiable se prépare depuis longtemps en lui. Retrouver son chemin, peut-être même emprunter comme si de rien n'était le transport en commun et se mêler aux somnambules qui, un peu comme moi avant, ne savaient pas à quoi s'en tenir. Honte de devoir mourir. Il faut pouvoir répondre à l'appel de la mort par le mot: présent.

Relisant les contributions stimulantes qui composent ce numéro, il s'en dégage qu'il n'est pas certain que le fait de communiquer une mauvaise nouvelle de cette nature soit susceptible d'apprentissage, de règles ou d'un savoir. À vrai dire, cette annonce requiert un non-savoir d'humanité: elle réclame l'art de la consolation, celui d'être né et d'en avoir mal. À cet égard, même le plus sombre diagnostic n'est pas une sentence. L'événement de la mort n'est pas une condamnation mais une condition. Certes, nous mourons encore dans les décors essoufflés de l'ère industrielle - reliés à des machines ou sous surveillance palliative -, mais cette technologie ou cet affairement normalisé et bureaucratique ne réussit pas à défigurer cette survenue impensable. Il faut accepter de subir la banalisation du fait de devoir soi-même mourir entre les rotations des quarts de nuit de syndiqués, dans les rumeurs lointaines des bavardages des personnels, en un mot, dans l'indifférence impeccable et professionnalisée du centre de soins. Peut-être est-ce cela l'horreur du mourir, bien au-delà de la souffrance des corps qui se désarticulent. Mourir sous surveillance, mais sans attention véritable à ce qui se dé-tresse dans ce qui a fait l'histoire du sujet. L'abandon véritable n'aurait-il lieu qu'au sein de la surveillance aguerrie, apparu à l'occasion du dispositif même de la sollicitude?

Écrire sur la mort implique la modestie d'accepter de ne pas savoir ce que l'on dit, tant pour nous cette conséquence 
physiologique paraît lointaine. Il ne saurait donc être question d'imaginer la rédaction d'un mode d'emploi, ou d'inclure une notice de type user friendly pour ceux qui ont la lourde tâche de dire à ceux qu'ils voient qu'ils sont promis à une mort imminente. Ces paroles restent à réinventer à chaque fois qu'un visage s'interroge dans l'attente. Elles impliquent une compétence plus haute que celle de l'établissement d'un diagnostic précis. Le boniment et aussi, il faut le dire quand même, l'éloge en pareilles circonstances sont tantôt menacés de ridicule ou simplement traversés par la duplicité sociale et reçue comme tel. Périlleuse parole des seuils.

À travers le riche éventail des contributions de nos auteurs, il est un bref texte qui retient mon attention, c'est celui qui pose la question du «droit des vivants à l'annonce de leur mort». Que voudrions-nous savoir que nous ne sachions déjà ? Qui croit encore à l'existence d'une médecine humaine? Le débat éthique sur le sens de la médecine moderne reste à faire. Comment ne pas percevoir le glissement d'une médecine qui «sauve la vie» vers celle qui « retarde la mort », pour finalement inscrire tout patient, contre son gré, dans un continuum pharmacie-économique?
Il ne suffit plus à l'homme moderne d'affronter la fin de sa vie dans une vacuité spirituelle sans précédent, il lui faut aussi prendre les dispositions légales pour échapper en mourant à l'appétit de connaissance de ceux qui prétendaient le soigner. Nous oscillons entre la perspective «constrictive» d'un acharnement thérapeutique ou son contraire, celle d'un funeste délaissement par suite d'opérations comptables d'allocation de ressources. L'accident statistique - le plus dangereux de tous à notre époque, - nous guette tout autant dans l'espérance de vie que dans l'expectative actuarielle de la possibilité de recevoir des soins.

Dans une optique plus large, il faudrait s'attarder à reconsidérer la valeur normative de concepts comme celui de la «qualité de vie ». Que signifie-t-elle dans l'espace ou dans l'univers du consommateur moyen? Il existe en filigrane à l'absurdité de l'existence de consommateur comme une jouissance de ne pas mourir en son temps, de s'épargner lamentablement... qui mène à la laideur du vieillissement insignifiant. Quel réajustement esthétique commande l'allongement de la vie et le maintien, par exemple, d'une vie sexuelle assistée par médication? Tout indique, telle la dispari- tion de la pudeur dans l'espace public - le fait de pouvoir tout dire et tout montrer que l'événement de mourir est ramené à sa seule expression biologique. Que signifie maintenant rester en vie lorsqu'on est célibataire, sans descendance? Comment concevoir une mort humaine qui ne serait ni annoncée ni racontée à personne?

\section{Bibliographie}

Legendre, P. (1996). La fabrique de l'homme occidental, Paris, Éditions Arte.

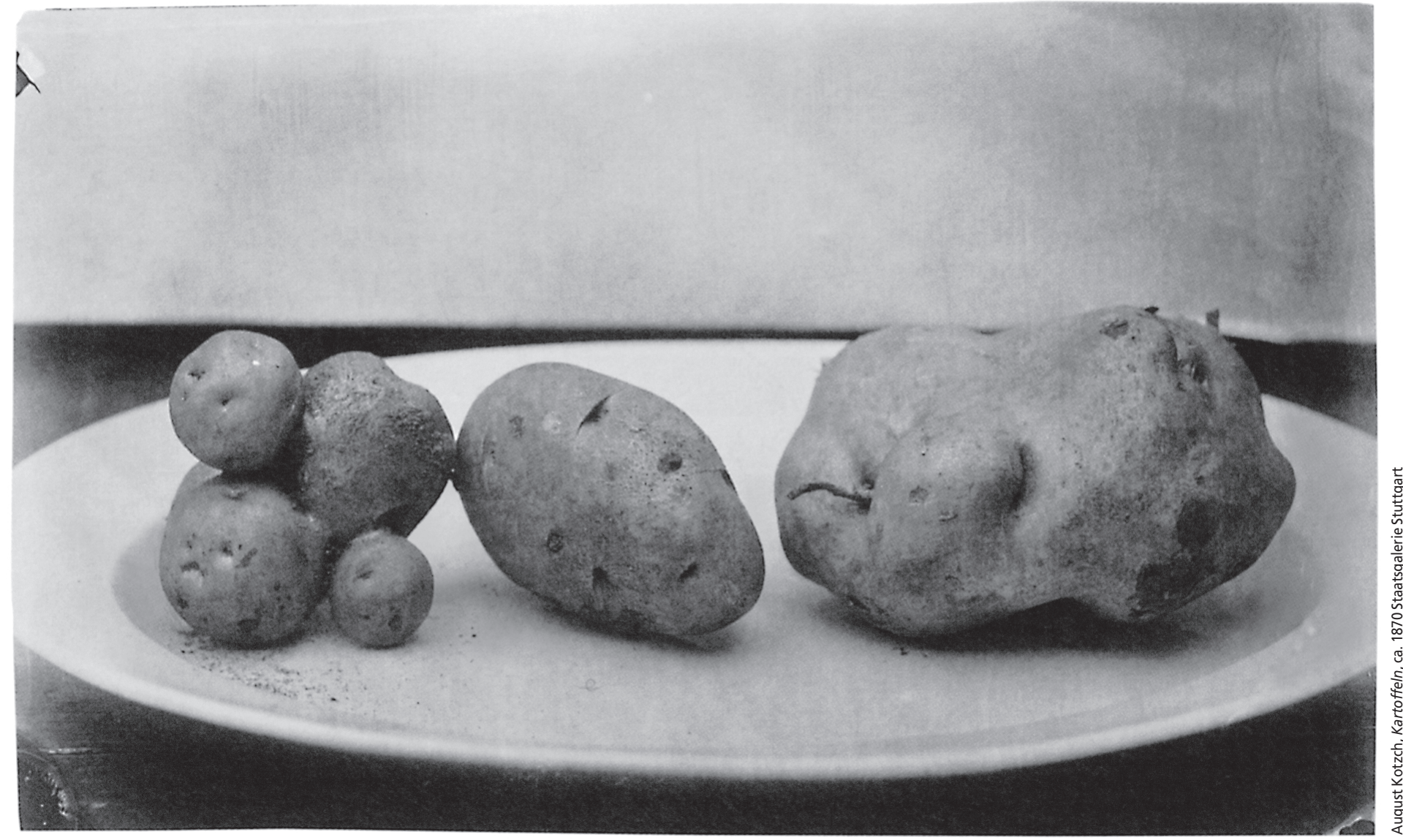

